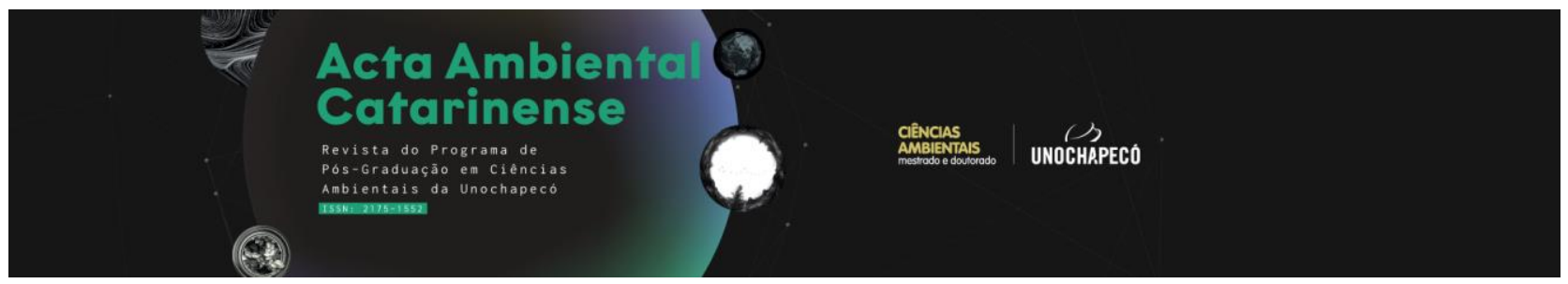

\title{
ORIGEM FITOGEOGRÁFICA, ESTRUTURA FILOGENÉTICA E SÍNDROMES DE DISPERSÃO DE ESPÉCIES ARBÓREAS EM FLORESTA OMBRÓFILA MISTA NO PLANALTO CATARINENSE
}

\author{
Larissa Cardoso Küster ${ }^{1}$ \\ Pedro Higuchi ${ }^{2}$ \\ Ana Carolina da Silva ${ }^{3}$ \\ Roni Djeison Ansolin ${ }^{4}$ \\ Marco Antônio Bento ${ }^{5}$ \\ Fernando Buzzi Júnior ${ }^{6}$ \\ Tiago de Souza Ferreira ${ }^{7}$
}

\begin{abstract}
Resumo
Os remanescentes florestais desempenham importantes funções ecossistêmicas, além de possuírem elevada diversidade residual. Um maior conhecimento ecológico destas áreas é fundamental para a sua conservação. Por isso, este estudo objetivou avaliar a fitogeografia, a estrutura filogenética e as síndromes de dispersão de espécies arbóreas de um fragmento de Floresta Ombrófila Mista no Planalto Catarinense, Brasil. As espécies amostradas no fragmento foram classificadas de acordo com sua origem fitogeográfica e síndrome de dispersão. A estruturação filogenética foi avaliada por meio dos valores de "Mean pairwise distance" (MPD) e de "Net Relatedness Index" (NRI). O gradiente ambiental foi analisado por meio de uma Análise de Componentes Principais. A influência do ambiente sobre a estruturação filogenética foi verificada por meio de um modelo linear pelo método dos Mínimos Quadrados Generalizados (GLS). As espécies tiveram a predominância de
\end{abstract}

\footnotetext{
${ }^{1}$ Departamento de Engenharia Florestal, CAV/UDESC, Av. Luiz de Camões, 2090, B. Conta Dinheiro, Cep. 88520000, Lages, SC, Brasil

${ }^{2}$ Departamento de Engenharia Florestal, CAV/UDESC, Av. Luiz de Camões, 2090, B. Conta Dinheiro, Cep. 88520000, Lages, SC, Brasil. Email: higuchip@gmail.com

${ }^{3}$ Departamento de Engenharia Florestal, CAV/UDESC, Av. Luiz de Camões, 2090, B. Conta Dinheiro, Cep. 88520000, Lages, SC, Brasil

${ }^{4}$ Departamento de Engenharia Florestal, CAV/UDESC, Av. Luiz de Camões, 2090, B. Conta Dinheiro, Cep. 88520000, Lages, SC, Brasil

${ }^{5}$ Departamento de Engenharia Florestal, CAV/UDESC, Av. Luiz de Camões, 2090, B. Conta Dinheiro, Cep. 88520000, Lages, SC, Brasil

${ }^{6}$ Departamento de Engenharia Florestal, CAV/UDESC, Av. Luiz de Camões, 2090, B. Conta Dinheiro, Cep. 88520000, Lages, SC, Brasil

${ }^{7}$ Departamento de Engenharia Florestal, CAV/UDESC, Av. Luiz de Camões, 2090, B. Conta Dinheiro, Cep. 88520000, Lages, SC, Brasil 
ampla distribuição $(62,3 \%)$ e zoocoria $(79,7 \%)$. A comunidade apresentou um padrão filogenético aleatório, sendo que o mesmo, em escala local, foi influenciado pela fertilidade do solo. Estes resultados sugeram que o perfil florístico de áreas de floresta com araucária foi influenciado por eventos históricos de migração das espécies a partir de diferentes origens, sendo que a fauna desempenhou um importante papel neste processo.

Palavras-chave: Floresta Ombrófila Mista; biogeografia histórica; filogeografia.

\begin{abstract}
The forest remnants perform important ecosystem functions and have a high residual diversity. A more in-depth ecological knowledge of these areas is fundamental for their conservation. Therefore, this study aimed to evaluate the phytogeography, phylogenetic structure, and dispersal syndromes of tree species in an Araucaria Forest fragment in the "Planalto Catarinense" region, Brazil. The sampled species in the fragment were classified according to their phytogeographic origin and dispersal syndrome. The phylogenetic structure was evaluated through the values of Mean Pairwise Distance (MPD) and Net Relatedness Index (NRI). The environmental gradient was analyzed by Principal Component Analysis. The environmental effect on phylogenetic structure was determined by Generalized Least Square models. The species had the predominance of wide geographic distribution (62.3\%) and zoochory (79.7\%). The community showed a random phylogenetic pattern, that was affected by soil fertility at the local scale. These results suggest that the floristic profile of Araucaria Forest areas was determined by historical events of migration of species from different origins, and the fauna played an important role in this process.
\end{abstract}

Keywords: Araucaria Forest; historical biogeography; phylogeography

\section{INTRODUÇÃ̃o}

Dentre as florestas brasileiras, a Floresta Ombrófila Mista (FOM) ou Floresta com Araucárias, que faz parte da Floresta Atlântica em sua porção subtropical (OLIVEIRA-FILHO et al., 2015), é caracterizada pelo predomínio de Araucaria angustifolia (Bertol.) Kuntze e pela mistura de espécies arbóreas com diferentes origens fitogeográficas (DUARTE et al., 2014). Devido ao histórico de exploração predatória e avanço de atividades agropecuárias, os remanescentes encontram-se atualmente em diferentes níveis de fragmentação (RIBEIRO et al., 2009).

Estudos pioneiros sobre a origem da flora da região Sul do Brasil foram realizados Vol. 18, N. 01 (2021) 
por Rambo (1951), que definiu, para o Rio Grande do Sul (RS), duas principais rotas de migração: a Leste, pela "Porta de Torres" (espécies provenientes do corredor de espécies tropicais atlânticas) e a Noroeste, pelo "Alto Uruguai" (espécies estacionais). Rambo (1953) citou que a Floresta Ombrófila Mista, localizada no planalto entre a Floresta Estacional Decidual, a oeste, e a Floresta Ombrófila Densa, a leste, é constituída por espécies de origem nas montanhas do Brasil Central, nos Andes e na flora austral-antártica, possuindo elementos anteriores aos que migraram pela floresta atlântica e estacional. Mais recentemente, alguns trabalhos sobre a origem fitogeográfica de espécies foram realizados em fragmentos de Floresta Estacional Decidual (FED) no RS (e.g. JARENKOW e WAECHTER, 2001; SÜHS et al., 2010), no entanto, com poucos sendo realizados em áreas de FOM (e.g., GONÇALVES e SOUZA, 2014).

Além dos estudos históricos da origem fitogeográfica, para maior compreensão do conjunto de espécies arbóreas de uma comunidade e a relação entre elas, abordagens filogenéticas podem ser relevantes. Considerando que as interações interespecíficas das espécies são mediadas por seus fenótipos, no caso de os mesmos apresentarem sinal filogenético, supõem-se que a estrutura filogenética de uma comunidade também possa indicar as interações ecológicas existentes (KRAFT et al., 2015). Desta forma, padrões não aleatórios, como o agrupamento ou sobredispersão filogenética, podem estar associados a fatores ecológicos, como filtros ambientais e interações interespecíficas, se os traços funcionais apresentarem sinal filogenético (SWENSON, 2012). Além disso, a estrutura filogenética de uma comunidade também pode refletir processos macroevolucionários, como por exemplo, aqueles que definem o conjunto regional de espécies (GERHOLD et al., 2015). No que se refere à estrutura filogenética de comunidades, apenas mais recentemente foram publicadas pesquisas sobre as florestas da região Sul do Brasil, destacando-se o trabalho de Duarte et al. (2014), que demonstraram a predominância de sobredispersão filogenética para comunidades da Floresta Ombrófila Mista. Em escala local, dentre as variáveis ambientais, os atributos dos solos e ambiente lumínico têm o potencial de desempenhar grande influência sobre a estrutura taxonômica (VALLADARES et al., 2016; NETTESHEIM et al., 2019), funcional (KERN et al., 2013; BECKNELL e POWERS, 2014) e filogenética de comunidades de espécies arbóreas (JIN et al. 2015; MORO et al., 2015). 
Contribuindo para uma melhor compreensão sobre o histórico da formação da FOM, estão os estudos que investigam as síndromes de dispersão das espécies, visto que a dispersão das espécies está diretamente associada à forma de colonização das áreas, influenciando, assim, a organização florísticaestrutural do componente arbóreo. Estudos realizados na região Sul do Brasil (e.g. ALMEIDA et al., 2008) têm demonstrado a importância da fauna para o processo de dispersão, uma vez que a síndrome predominante é a zoocórica.

Assim, considerando que pesquisas sobre a origem fitogeográfica, estrutura filogenética de comunidades e síndromes de dispersão das espécies são fundamentais, pois permitem inferências sobre o processo histórico de formação desta floresta e a ecologia das espécies (DUARTE et al. 2014; GONÇALVES e SOUZA, 2014), o objetivo desse estudo foi analisar: a origem fitogeográfica, a estrutura filogenética e as síndromes de dispersão das espécies arbóreas encontradas em um fragmento de Floresta Ombrófila Mista, na região do Planalto Sul Catarinense. Testou-se que a hipótese de que: i) a flora arbórea do remanescente florestal avaliado é caracterizada por possuir elementos taxonômicos com diferentes origens fitogeográficas, com a fauna sendo o principal vetor na dispersão de propágulos; ii) a

\section{MAterial e Métodos}

\section{1 Área de Estudo}

Foi estudado um fragmento natural de Floresta Ombrófila Mista inserido na região da Coxilha Rica em Lages, SC, inventariado por Ansolin et al. (2016). A região pode ser considerada como sendo caracterizada por um mosaico de formações vegetacionais, composto, predominantemente por campos de altitude e "capões de mata" de Floresta Ombrófila Mista. O fragmento florestal avaliado apresenta uma área aproximada de 18 ha e encontra-se nas coordenadas de latitude $28^{\circ} 07^{\prime} 15^{\prime}$ 'S e longitude $50^{\circ} 18^{\prime} 25^{\prime}$ ' O, com altitude de $1.050 \mathrm{~m}$. O clima é $\mathrm{Cfb}$ (temperado/subtropical úmido, com verões amenos), segundo Köppen, a precipitação anual média é de $1.682,80 \mathrm{~mm}$, bem distribuídas no ano, e a temperatura anual média é de $15,9^{\circ} \mathrm{C}$, de acordo com dados obtidos da estação meteorológica de Lages, administrada pelo Centro de Informações de Recursos Ambientais e de Hidrometeorologia de Santa Catarina (EPAGRI/CIRAM). A partir de observações no campo, foi possível constatar que o fragmento não apresenta indícios de perturbações recentes e encontrase protegido e em avançado estádio sucessional, embora o histórico de toda a Vol. 18, N. 01 (2021) 
região é de cortes seletivos, principalmente nas décadas de 1950 até 1970 . No interior do fragmento há cursos de água de pequeno porte e, conforme tradagens realizadas nas parcelas amostrais, solos que podem ser classificados como Gleissolos e Cambissolos.

\subsection{Desenho Amostral}

No presente estudo, foram utilizados os dados vegetacionais e ambientais coletados por Ansolin et al. (2016). Para o levantamento da composição florística, os autores supracitados alocaram, de forma sistemáticaestratificada, 50 parcelas de 10x20 m (200 $\mathrm{m}^{2}$ ), totalizando 1 ha de área amostrada. As parcelas foram distribuídas em estratos, com o propósito de contemplar os diferentes setores ambientais do fragmento avaliado, com parcelas distantes $5 \mathrm{~m}$ entre si (ver ANSOLIN et al., 2016). Em cada parcela, os indivíduos arbóreos vivos que apresentaram CAP (circunferência à altura do peito, medida a $1,30 \mathrm{~m}$ do solo) igual ou superior a $15,7 \mathrm{~cm}$ foram identificados. As identificações foram realizadas por meio de literatura (SOBRAL et al., 2013) e consulta a especialistas e as espécies de angiospermas foram classificadas nas famílias de acordo com o sistema APG IV (APG, 2016). Da mesma forma, para as mesmas parcelas, Ansolin et al. (2016) determinaram a cobertura de dossel, por meio de um densiômetro esférico, caracterizaram o relevo, com o auxílio de um clinômetro, e as propriedades físico-químicas dos solos, a partir de amostras compostas dos mesmos.

Para o presente estudo, foram determinadas as origens fitogeográficas das espécies por meio da classificação dessas em categoria de contingente de migração, utilizando-se a metodologia proposta por Jarenkow e Waechter (2001), que consideram em seu estudo os corredores de migração do Atlântico ou corredor leste (ATL) e das bacias dos rios Paraná e Uruguai ou corredor oeste (BPU). Nesse caso, os autores excluíram os Pinhais (Floresta Ombrófila Mista). No presente trabalho foi considerado, também, a Floresta Ombrófila Mista como uma origem fitogeográfica, já que o estudo foi realizado nesta mesma formação florestal. As espécies que tinham origem em duas ou mais formações foram classificadas como espécie de ampla distribuição (EAD).

A síndrome de dispersão de propágulos primária de cada espécie encontrada no fragmento foi definida de acordo com Van der Pijl (1982), em que: i) espécies de frutos carnosos que evidenciam a dispersão por animais, foram consideradas como de dispersão zoocórica; ii) frutos ou sementes com alas e mecanismos para flutuação, foram definidos como de dispersão anemocórica; iii) frutos tipo cápsula deiscente que, ao se abrir, liberam as sementes por um rápido movimento, foram considerados de dispersão 
autocórica. A atribuição dessas síndromes para as espécies observadas no presente estudo se deu por observações dos diásporos quando disponíveis no campo e, no caso de não visualização dos diásporos no campo, por consultas em literatura (ALMEIDA et al., 2008).

\subsection{Análise dos dados}

A partir o conjunto de espécies presentes no Banco de Dados do Laboratório de Dendrologia da Universidade do Estado de Santa

Catarina (https://figshare.com/s/9735ebd1691d7a90f0 5f), construído por meio de levantamentos fitossociológicos realizados na região, foi criada uma árvore filogenética, representando o pool regional de espécies que podem potencialmente ocorrer na área. Para isto foi utilizado o PHYLOMATIC 3, disponível em <http://phylodiversity.net/phylomatic/>, utilizando a "super-árvore" filogenética R20160415.new, disponibilizada por Gastauer e Meira-Neto (2017), que é baseada na topologia proposta pela APG IV (APG, 2016). A árvore filogenética criada foi importada para o programa Phylocom 4.1. (WEBB et al., 2008), no qual os comprimentos dos "ramos" foram ajustados utilizando o algoritmo BLADJ, em função das idades dos clados, em milhões de anos, fornecidos por Gastauer e Meira Neto (2017)
(ages_magallon_PL.txt), baseado no trabalho de Magallón et al. (2015).

Ainda por meio do Phylocom, foram determinados o valor da Distância Filogenética Média, (MPD, "Mean Phylogenetic Distance") e o Índice de Parentesco Líquido (NRI, "Net relatedness index") (WEBB, 2000) utilizando, para isto, uma matriz de abundância das espécies nas parcelas. O valor de MPD indica o valor médio da distância filogenética entre todas as combinações de pares de espécies e o NRI representa uma medida de dispersão filogenética. Para verificar a existência de estruturação filogenética, o valor de MPD observado foi comparado com o valor de MPD esperado para uma comunidade completamente aleatória, calculado a partir 999 simulações, por meio de um modelo nulo que faz a aleatorização dos táxons ao longo da árvore filogenética ("Phylogeny Pool”). Nesse método, se o valor de NRI for positivo e o MPD observado for inferior aos MPDs simulados por, pelo menos, 975 vezes, considera-se a ocorrência de agrupamento filogenético $(p \leq 0,05)$. Se o valor de NRI for negativo e, por, pelo menos, 975 vezes, o MPD observado for superior aos MPDs simulados, considera-se a ocorrência de dispersão filogenética $(p \leq 0,05)$ (WEBB, 2000). Dicksonia sellowiana Hook, Araucaria angustifolia e Podocarpus lambertii Klotzsch 
ex Endl. não foram considerados na análise por se tratar de taxa distantes filogeneticamente de outras espécies, o que poderia representar uma fonte de viés na análise filogenética.

Para analisar a influência do ambiente sobre a estrutura filogenética em escala local no fragmento florestal, foram utilizados os dados ambientais coletados em cada uma das 50 parcelas por Ansolin et al. (2016). Com o propósito de se identificar os principais gradientes ambientais, estes dados foram ordenados por meio de uma Análise de Componentes Principais (PCA), sendo as variáveis de maior contribuição para a ordenação plotadas na forma de vetores. Os scores das parcelas ao longo do Eixo 1 e 2 da PCA, que são os mais explicativos, foram utilizados como variáveis explicativas da estruturação filogenética (NRI). Foram ajustados modelos lineares utilizando $\mathrm{O}$ método dos Mínimos Quadrados Generalizados (GLS), de forma que a estrutura espacial foi incorporada testando diferentes tipos de correlações (exponencial, gaussiana, linear, quadrático racional e esférico). No modelo, as variáveis explicativas foram testadas de forma isoladas $(\mathrm{NRI} \sim$ Scores PCA1 e NRI $\sim$ Scores PCA2) e de forma combinada (NRI $\sim$ Scores PCA1 + Scores PCA2). A seleção de ajuste se deu pelo menor valor de AIC (Critério de Seleção de
Akaike). O ajuste selecionado foi validado pela análise de resíduos (distribuição, tendência, independência). A modelagem foi realizada por meio da linguagem de programação estatística R (R CORE TEAM, 2019).

\section{RESUltados}

Foram encontradas 69 espécies arbóreas pertencentes a 46 gêneros e 28 famílias. De acordo com a árvore filogenética (Figura 1), excluindo-se Dicksonia sellowiana, Araucaria angustifolia e Podocarpus lambertii, as espécies mais basais foram as Magnoliídeas Drimys brasiliensis Miers (Winteraceae), Cinnamodendron dinissi Schwanke (Canellaceae), Persea major (Meisn.) L. E. Kopp, Cinnamomum amoemum (Nees) Kosterm., Ocotea pulchella (Nees) Mez, Nectandra megapotamica (Spreng.) Mez (Lauraceae) e Annona rugulosa (Schltdl.) H.Rainer (Annonaceae). O clado de maior riqueza foi Rosídeas, com 43 espécies, representando $62,3 \%$ das espécies amostradas, sendo Myrtaceae a família mais representativa (19), seguida por Asterídeas, com 17 espécies. A distância média entre os táxons, representado pelo valor de MPD, foi de 214 milhões de anos, com NRI de 0,72. Como o valor de NRI foi positivo e, por 785 vezes, o MPD observado foi inferior aos MPDs simulados, cuja média foi de 219 
milhões de anos, assume-se que as espécies encontradas no fragmento representam um subconjunto aleatório do pool regional ( $p>$ $0,05)$. Considerando os valores observados nas parcelas, o valor de MPD e NRI médio foram, respectivamente, de 194,13 e 0,48, sendo que a maior parte das mesmas (43) também apresentou uma estruturação filogenética completamente aleatória.

Os dois primeiros eixos da PCA explicaram, respectivamente, 27,1 e $19,8 \%$ da inércia total dos dados ambientais (Figura 2a).

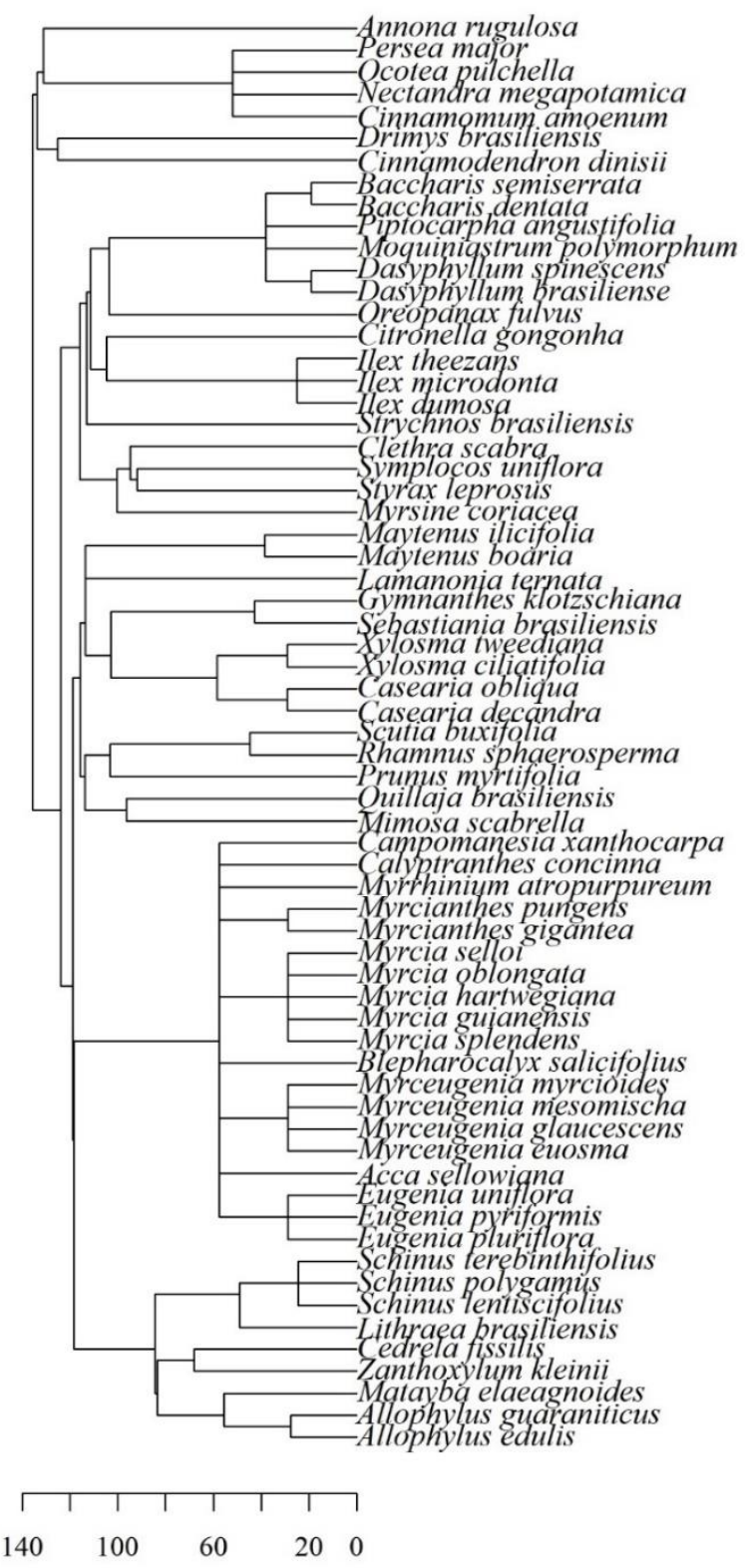

Figura 1. Árvore filogenética construída a partir das espécies arbóreas amostradas em um fragmento de Floresta Ombrófila Mista na região do Planalto Sul Catarinense. Escala em milhões de anos. 
Dentre as variáveis de maior contribuição, as três que apresentaram os maiores valores de loadings em relação ao eixo 1 foram saturação por bases (SatBas), teor de cálcio (Ca) e pH. Em relação ao eixo 2, foram teor de matéria orgânica (MO), declividade (dec) e desnível topográfico (desn). Desta forma, infere-se que os principais gradientes ambientais na área estudada estão relacionados, principalmente, à fertilidade do solo (eixo 1) e ao relevo (eixo 2). Após ajustes realizados para análise da influência destes gradientes ambientais (eixos 1 e 2 da PCA) sobre a estrutura filogenética nas parcelas (NRI), o modelo que apresentou menor valor de AIC (159) foi o que considerou apenas o eixo 1 da PCA como variável explicativa (Figura 2b) e a incorporação da estruturação espacial por meio de uma correlação do tipo exponencial. Neste modelo, foi possível identificar que a fertilidade do solo tendeu a influenciar a estrutura filogenética da comunidade ( $p=$ 0,0883). Conforme observado na Figura 2b, quanto maior a maior fertilidade, maior foi o valor de NRI, sugerindo que nos locais de solos menos férteis os indivíduos arbóreos são filogeneticamente mais distantes entre si do que nos locais de maior fertilidade.

Na classificação das espécies na origem fitogeográfica, as espécies com ampla distribuição geográfica obtiveram maior incidência $(62,3 \%)$, seguidas das espécies da
FOM $(24,6 \%)$, essas últimas representadas por Acca sellowiana (O. Berg) Burret, Araucaria angustifolia, Cinnamodendron dinisii, Cinnamomum amoenum, Clethra scabra Pers., Drimys brasiliensis, Eugenia pluriflora DC., Ilex microdonta Reissek, Maytenus boaria Molina, Maytenus ilicifolia Mart. ex Reissek, Myrceugenia euosma (O. Berg) D. Legrand, Myrceugenia mesomischa (Burret) D. Legrand et Kausel, Oreopanax fulvus Marchal, Piptocarpha angustifolia Dusén ex Malme, Quillaja brasiliensis (A. St.-Hil. \& Tul.) Mart., Schinus lentiscifolius Marchand e Zanthoxylum kleinii (R. S. Cowan) P. G. Waterman. Em menor proporção, estiveram as espécies originadas da bacia do rio Paraná e Uruguai $(13,0 \%)$, representadas por Allophylus guaraniticus (A. St.-Hil.) Radlk., Calyptranthes concinna DC., Casearia decandra Jacq., Dasyphyllum brasiliense (Spreng.) Cabrera, Dasyphyllum spinescens (Less.) Cabrera, Myrcia oblongata DC., Myrcianthes pungens (O. Berg) D. Legrand, Scutia buxifolia Reissek e Xylosma tweediana (Clos) Eichler. Não foi encontrada espécie com origem exclusiva do continente Atlântico. 

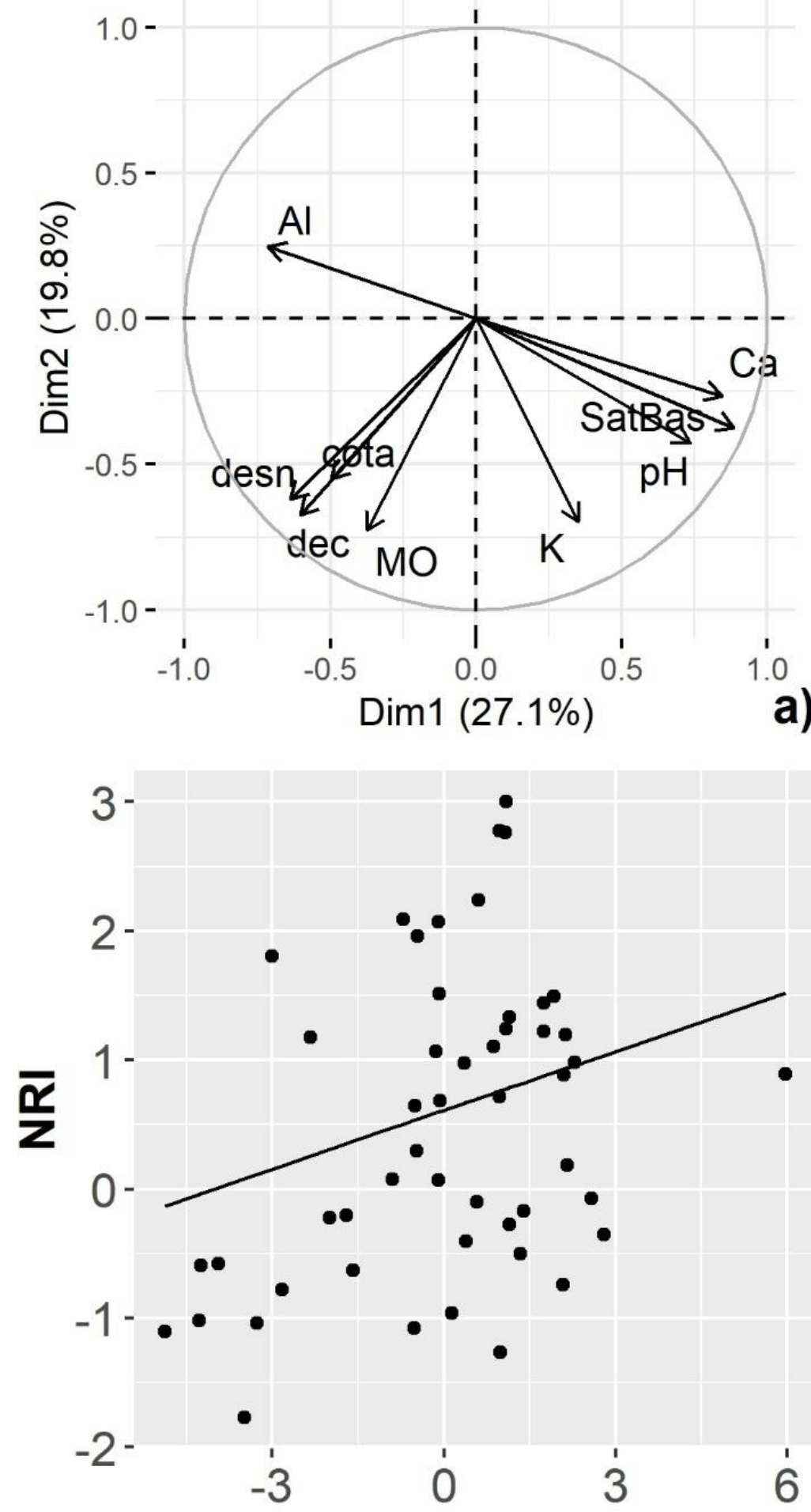

Scores PCA1

Em relação à síndrome de dispersão, $79,7 \%$ das espécies foram classificadas com dispersão zoocórica, 15,9\% anemocórica e
Figura 2. Análise de Componentes Principais (PCA), com variáveis ambientais de maior contribuição indicadas na forma de vetores (a) (Al a) - teor de alumínio, desn - desnível do relevo, dec - declividade do relevo, cota - cota altimétrica, $\mathrm{MO}-$ teor de matéria orgânica, $\mathrm{K}$ - teor de potássio, $\quad \mathrm{pH} \quad-$ potencial hidrogeniônico, SatBas - saturação por bases, $\mathrm{Ca}$ - teor de cálcio) para um fragmento de Floresta Ombrófila Mista na região do Planalto Sul Catarinense. Influência do gradiente ambiental sintetizado pelo Eixo1 da PCA (fertilidade) sobre a estrutura filogenética (NRI) (b). b)
4,4\% autocórica. As famílias Euphorbiaceae e Fabaceae apresentaram todas as espécies com síndrome de dispersão autocórica e 
Asteraceae, Clethraceae, Cunoniaceae, Dicksoniaceae, Meliaceae e Quillajaceae, todas as espécies com síndrome anemocórica. Para todas as categorias de origem fitogeográfica, as espécies foram predominantemente zoocóricas $(>70 \%)$, no entanto, a autocoria só ocorreu nas espécies de ampla distribuição (Figura 3).

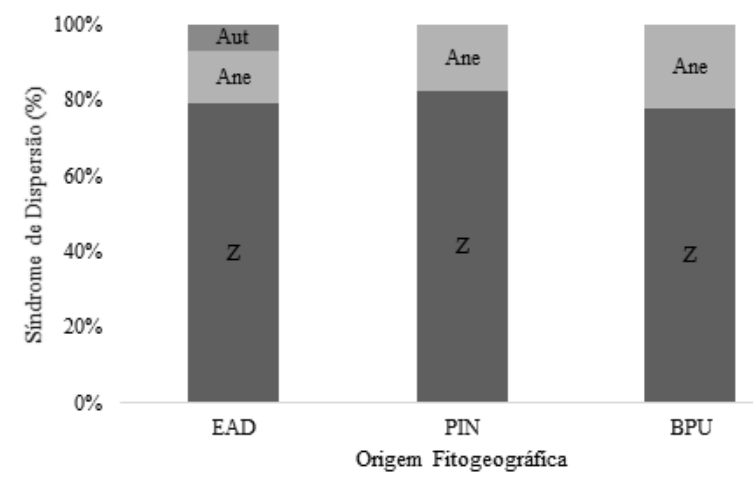

Figura 3. Proporções das síndromes de dispersão nas espécies das diferentes origens fitogeográficas encontradas em um fragmento de Floresta Ombrófila Mista na região do Planalto Sul Catarinense. EAD= espécies com ampla distribuição; PIN= espécies da Floresta Ombrófila Mista; BPU= espécies das bacias dos rios Paraná e Uruguai; Zoo= zoocóricas; Ane $=$ anemocóricas; Aut $=$ autocóricas.

\section{DISCUSSÃo}

O conjunto de espécies amostradas possui diferentes origens fitogeográficas. Dentre as espécies mais basais, enquanto Araucaria angustifolia, Drimys brasiliensis e Cinnamodendron dinissi têm suas origens ligada a flora gondwânica temperada, Lauraceae e Annoaceae estão associadas a migrações laurasianas (FIASCHI e PIRANI, 2009). No clado das Rosídeas, destaca-se o gênero Myrcia (Myrtaceae), de origem neotropical (SYTSMA et al., 2004). No clado Asterídeas, destacam-se as espécies pertencentes ao gênero cosmopolita Ilex, que já ocupava diferentes partes do globo terrestre desde o fim do Cretáceo, em torno de 65 milhões de anos atrás (CUÉNOUD et al., 2000).

Os elementos taxonômicos da área florestal estudada representaram um subconjunto completamente aleatório do pool regional de espécies da FOM. Este resultado difere do padrão observado por Duarte et al. (2014), em um estudo em escala regional, que observou, para a mesma fitofisionomia, a ocorrência de espécies filogeneticamente distantes entre si. Neste caso, o contraste de resultados se deve as diferenças metodológicas, relacionadas às escalas espaciais mais amplas, e à abrangência do pool de espécies contemplando todo do Domínio Atlântico, para os cálculos das métricas filogenéticas.

Apesar do padrão aleatório descrito acima, destaca-se que a fertilidade do solo influenciou a estruturação filogenética observada em escala local (parcelas). A presença de um número maior de clados, 
expresso pelo menor valor de NRI, em locais relativamente menos férteis, evidencia a presença de linhagens com históricos evolutivos distintos nestes ambientes. Por outro, as parcelas com solos mais férteis tenderam a apresentar menor diversidade filogenética. Estes resultados sugerem que na comunidade avaliada, os atributos funcionais que permitem melhores adaptações para o desenvolvimento em locais menos férteis não sejam filogeneticamente conservados (SANDER e WARDELL-JOHNSON, 2011) e, ou, que em condições de maior fertilidade a comunidade tenha tendência ser mais influenciada pelo processo de exclusão competitiva (CAVENDER-BARES et al. 2009). Isto demonstra a relevância das condições edáficas, como a fertilidade do solos, não apenas para a organização florístico-estrutural, como já demonstrado por Ansolin et al. (2016) na mesma área, mas também para a estrutura filogenética de comunidades, como já relatado por $\mathrm{Xu}$ et al. (2016) em uma floresta tropical na Ásia.

A predominância de espécies de ampla distribuição geográfica corrobora com a ideia de que a flora regional representa um resultado de processos de migração de espécies a partir de diferentes origens fitogeográficas (DUARTE et al., 2014). Já a elevada ocorrência de espécies de origem na FOM está relacionada à altitude do local, superior a $900 \mathrm{~m}$, e ao clima temperado, sendo a área estudada um fragmento típico de Floresta Ombrófila Mista (HIGUCHI et al., 2013; OLIVEIRA-FILHO et al., 2015). Sühs et al. (2010), em um estudo em de Floresta Estacional, porém, com alguns elementos da Floresta Ombrófila Mista, obtiveram resultados semelhantes quanto a espécies de ampla distribuição geográfica, porém, espécies provenientes da bacia dos rios Paraná e Uruguai e do contingente Atlântico obtiveram maior incidência do que espécies de origem na FOM. Esse padrão provavelmente foi encontrado porque a área estudada por eles possuía maior influência estacional do que mista, além da altitude de $375 \mathrm{~m}$ acima do nível do mar.

A relação entre a origem fitogeográfica com as rotas migratórias sugeridas por Rambo (1951) é evidenciada nesse estudo. Além do elevado número de espécies de origem na FOM (24,6\%), muitas espécies de origem nas bacias dos rios Paraná e Uruguai (13,0\%), quando comparado com a representatividade de origem atlântica, confirmam a ausência de barreira geográfica e climática entre o planalto da FOM e região oeste do estado. Assim, observa-se a influência expressiva de espécies características das florestas das bacias dos rios Paraná e Uruguai. Não foi encontrada espécie de origem exclusiva atlântica, o que reforça a ideia de um relativo baixo compartilhamento 
florístico entre a FOM e a Floresta Ombrófila Densa (FOD) (GONÇALVES e SOUZA, 2014).

A síndrome de dispersão zoocórica é comum em florestas subtropicais no Brasil (e.g. ALMEIDA et al., 2008). A alta proporção de espécies deste grupo confirma a importância da fauna para a dispersão de propágulos e, consequentemente, para a manutenção do fluxo gênico das florestas, de forma que a redução das populações de animais pode ter implicações diretas na extinção local ou diminuição de espécies zoocóricas (ALMEIDA et al., 2008).

As espécies das categorias de origem fitogeográfica não apresentaram grande variação no percentual referente à representatividade da síndrome de dispersão zoocórica, com a predominância dessa síndrome em todos os casos. Porém, o fato da autocoria estar presente somente para as espécies de ampla distribuição não era esperado, devido à limitação da distância de dispersão, uma vez que, supostamente, os propágulos teriam baixa capacidade de deslocamento em longa distância.

Conclui-se que a flora arborescente do fragmento estudado é formada por elementos taxonômicos de diferentes origens fitogeográficas, tendo a fauna como principal vetor de dispersão. Além disso, as espécies presentes representam um subconjunto completamente aleatório do conjunto regional avaliado da Floresta Ombrófila Mista. Em escala local, ambientes mais férteis apresentam indivíduos arbóreos com maior proximidade filogenética do que aqueles menos férteis.

\section{Agradecimentos}

À Lúcia B. Ávila, pela disponibilidade da área de estudo, e ao CNPq, pela concessão de bolsa de produtividade em pesquisa ao segundo e terceiro autores.

\section{REFERÊNCIAS}

ALMEIDA SR, WATZLAWICK LF, MYSZKA E. Florística e síndromes de dispersão de um remanescente de Floresta Ombrófila Mista em sistema faxinal. Ambiência, Guarapuava, v. 4, n. 2, p. 289297, 2008.

ANSOLIN RD, SILVA AC, HIGUCHI P, KUSTER LC, FERREIRA TS, BUZZI JUNIOR F, BENTO MA, AGUIAR MD, CRUZ AP. Heterogeneidade ambiental e variação florístico-estrutural em um fragmento de floresta com araucária na Coxilha Rica, SC. Ciência Florestal, Santa Maria, v. 26, n. 4, p. 1201-1210, 2016. 
APG. An update of the Angiosperm Phylogeny Group classification for the orders and families of flowering plants: APG IV. Botanical Journal of the Linnean Society, London, v. 181, n. 1, p. 1-20, 2016.

BECKNELL JM, POWERS JS, Stand age and soils as drivers of plant functional traits and aboveground biomass in secondary tropical dry forest. Canadian Journal of Forest Research, v. 44, n. 6, p. 604-613, 2014.

CAVENDER-BARES J, KOZAK KH, FINE PVA, KEMBEL SW. The merging of community ecology and phylogenetic biology. Ecology Letters, Hoboken, v. 12, n. 7, p. 693-715, 2009.

CUÉNOUD P, MARTINEZ MADP, LOIZEAU PA, SPICHIGER R, ANDREWS S, MANEN JF. Molecular phylogeny and biogeography of the genus Ilex L. (Aquifoliaceae). Annals of Botany, Oxford, v. 85, n. 1, p. 111-122, 2000.

DUARTE LS, BERGAMIN RS, MARCILIOSILVA V, SEGER GDS, MARQUES MCM. Phylobetadiversity among forest types in the brazilian Atlantic Forest Complex. PloS ONE, São Francisco, v. 9, n. 8, p. e105043, 2014.
FIASCHI P, PIRANI JR. Review of plant biogeographic studies in Brazil. Journal of Systematics and Evolution, Hoboken, v. 47, n. 5, p. 477-496, 2009.

GASTAUER M, MEIRA NETO JAA. Updated angiosperm family tree for analyzing phylogenetic diversity and community structure. Acta Botanica Brasilica, v. 31, n. 2, p. 191-198, 2017.

GERHOLD P, CAHILL JR JF, WINTER M, BARTISH IV, PRINZING, A. Phylogenetic patterns are not proxies of community assembly mechanisms (they are far better). Functional Ecology, London, v. 29, n. 5, p. 600-614, 2015.

GONÇALVES ET, SOUZA AF. Floristic variation in ecotonal areas: patterns, determinants and biogeographic origins of subtropical forests in South America. Austral Ecology, Hoboken, v. 39, n. 1, p. 122-134, 2014.

JARENKOW JA, WAECHTER JL. Composição, estrutura e relações florísticas do componente arbóreo de uma floresta estacional no Rio Grande do Sul, Brasil. Revista Brasileira de Botânica, São Paulo, v. 24, n. 3, p. 263-272, 2001. 
JIN Y, QIAN H, YU M. Phylogenetic structure of tree species across different life stages from seedlings to canopy trees in a subtropical evergreen broad-leaved forest. PloS one, v. 10, n. 6, 2015.

KERN CC, MONTGOMERY RA, REICH PB, STRONG TF. Canopy gap size influences niche partitioning of the ground-layer plant community in a northern temperate forest. Journal of Plant Ecology, v. 6, n. 1, p. 101112, 2013.

KRAFT NJB, ADLER PB, GODOY O, JAMES EC, FULLER S, LEVINE, JM. Community assembly, coexistence and the environmental filtering metaphor. Functional Ecology, London, v. 29, n. 5, p. 592-599, 2015.

MAGALLÓN S, GÓMEZ-ACEVEDO S, SÁNCHEZ-REYES LL, HERNÁNDEZHERNÁNDEZ T. A metacalibrated time-tree documents the early rise of flowering plant phylogenetic diversity. New Phytologist, v. 207, n. 2, p. 437-453, 2015.

HIGUCHI P, SILVA AC, BUDKE JC, MANTOVANI A, BORTOLUZZI RLC, ZIGER AA. Influência do clima e de rotas migratórias de espécies arbóreas sobre o padrão fitogeográfico de florestas na região sul do brasil. Ciênc. Florest., v. 23, n. 4, p. 539-553, 2013.

MORO, MF, SILVA IA, ARAUJO FS, LUGHADHA EN, MEAGHER TR, MARTINS FR. The role of edaphic environment and climate in structuring phylogenetic pattern in seasonally dry tropical plant communities. PLoS One, v. 10, n. 3, p. e0119166, 2015.

NETTESHEIM FC, GARBIN ML, PEREIRA MG , ARAUJO DSD. Local environmental controls of Atlantic Forest tree community assembly on a coastal continental island in southeastern Brazil. Acta Bot. Bras. v. 33, n.1, p. 88-96, 2019.

OLIVEIRA-FILHO AT, BUDKE JC, JARENKOW JA, EISENLOHR PV, NEVES DR. Delving into the variations in tree species composition and richness across South American subtropical Atlantic and Pampean forests. Journal of plant ecology, v. 8, n. 3, p. 242-260, 2015.

R CORE TEAM. R: A Language and Environment for Statistical Computing. R Foundation for Statistical Computing, Vienna, Austria, 2019. 
RAMBO B. A imigração da selva higrófila no Rio Grande do Sul. Anais Botânicos do Herbário Barbosa Rodrigues, Itajaí, v. 3, s/n, p. 55-91, 1951.

RAMBO B. História da flora do planalto riograndense. Anais Botânicos do Herbário Barbosa Rodrigues, Itajaí, v. 5, s/n, p. 185232, 1953.

RIBEIRO MC, METZGER JP, MARTENSEN AC, PONZONI FJ, HIROTA MM. The Brazilian Atlantic Forest: How much is left, and how is the remaining forest distributed? Implications for conservation. Biological Conservation, Amsterdam, v. 142, n. 6, p. 1141-1153, 2009.

SANDER J, WARDELL-JONHSON G. Impacts of soil fertility on species and phylogenetic turnover in the high - rainfall zone of the Southwest Australian global biodiversity hotspot. Plant and Soil, v. 345, p. 103-124, 2011.

SOBRAL M, JARENKOW JA, BRACK P, IRGANG B, LAROCCA J, RODRIGUES RS. Flora Arbórea e Arborescente do Rio Grande do Sul, Brasil. Editora RiMA, São Carlos, 362p, 2013.
SÜHS RB, PUTZKE J, BUDKE JC. Relações florístico-geográficas na estrutura de uma floresta na região central do Rio Grande do Sul, Brasil. Floresta, Curitiba, v. 40, n. 3, p. 635-646, 2010.

SYTSMA KJ, LITT A, ZJHRA ML, PIRES JC, NEPOKROEFF M, CONTI E, WALKER J, WILSON PG. Clades, clocks, and continents: historical and biogeographical analysis of Myrtaceae, Vochysiaceae, and relatives in the southern hemisphere. International Journal of Plant Sciences, Chicago, v. 165, n. S4, p. S85-S105, 2004.

SWENSON NG. The assembly of tropical tree communities-the advances and shortcomings of phylogenetic and functional trait analyses. Ecography, Hoboken, v. 36, n. 3, p. 264-276, 2013

VALLADARES F, LAANISTO L, NIINEMETS Ü, ZAVALA MA. Shedding light on shade: ecological perspectives of understorey plant life. Plant Ecology \& Diversity, v. 9, n. 3, p. 237-251, 2016.

\section{VAN DER PIJL L. Principles of dispersal in} higher plants. Berlin: Springer-Verlarg, 1982. $161 \mathrm{p}$.

XU W, CI X, SONG C, HE T, ZHANG W, LI

Q, LI J. Soil phosphorus heterogeneity 
promotes tree species diversity and phylogenetic clustering in a tropical seasonal rainforest. Ecology and Evolution, Hoboken, v. 16, n. 24, p. 8719-8726, 2016.

WEBB CO. Exploring the phylogenetic structure of ecological communities: an example for rain forest trees. The American Naturalist, Chicago, v. 156, n. 2, p. 145-155, 2000.

WEBB CO, ACKERLY DD, KEMBEL SW. Phylocom: software for the analysis of phylogenetic community structure and trait evolution. Bioinformatics, Oxford, v. 24, n. 18, p. 2098-2100, 2008. 